\title{
Electricity Generation Characteristics of Energy-Harvesting System with Piezoelectric Element Using Mechanical-Acoustic Coupling
}

\author{
Hirotarou Tsuchiya, Hiroyuki Moriyama, and Satoru Iwamori \\ Course of Science and Technology, Graduate School of Tokai University, 4-1-1 Kitakaname, Hiratsuka, Kanagawa, Japan \\ Correspondence should be addressed to Hiroyuki Moriyama; moriyama@keyaki.cc.u-tokai.ac.jp
}

Received 1 March 2016; Revised 23 August 2016; Accepted 31 August 2016

Academic Editor: Mohammad Tawfik

Copyright (C) 2016 Hirotarou Tsuchiya et al. This is an open access article distributed under the Creative Commons Attribution License, which permits unrestricted use, distribution, and reproduction in any medium, provided the original work is properly cited.

\begin{abstract}
This paper describes the electricity generation characteristics of a new energy-harvesting system with piezoelectric elements. The proposed system is composed of a rigid cylinder and thin plates at both ends. The piezoelectric elements are installed at the centers of both plates, and one side of each plate is subjected to a harmonic point force. In this system, vibration energy is converted into electrical energy via electromechanical coupling between the plate vibration and piezoelectric effect. In addition, the plate vibration excited by the point force induces a self-sustained vibration at the other plate via mechanical-acoustic coupling between the plate vibrations and an internal sound field into the cylindrical enclosure. Therefore, the electricity generation characteristics should be considered as an electromechanical-acoustic coupling problem. The characteristics are estimated theoretically and experimentally from the electric power in the electricity generation, the mechanical power supplied to the plate, and the electricity generation efficiency that is derived from the ratio of both power. In particular, the electricity generation efficiency is one of the most appropriate factors to evaluate a performance of electricity generation systems. Thus, the effect of mechanical-acoustic coupling is principally evaluated by examining the electricity generation efficiency.
\end{abstract}

\section{Introduction}

To deal with depletion of fossil fuels and to materialize a low-carbon society, not only the improvement of energy saving technologies but also the creation of new energy sources has been attempted in a lot of studies. Scavenging untapped vibration energy and converting it into usable electric energy via piezoelectric materials has attracted considerable attention and has been regarded as one of new energy sources [1]. Typical energy harvesters adopt a simple cantilever configuration to generate electric energy via piezoelectric materials, which are attached to or embedded in host structures, and the behavior is governed by electromechanical coupling phenomena.

In general, the flexural stiffness of a beam structure is considerably intensified in comparison with that of a piezoelectric element, so that most of the strain energy is stored in the beam structure. To enhance the conversion efficiency, two methods have been adopted in many cases: the optimization of piezoelectric element placement and the use of a large element or many elements. To further improve the conversion efficiency, mechanical impedance matching method, which was derived from using spacers between the piezoelectric element and beam structure and tuning for the size of the piezoelectric element, was proposed [2]. These structural vibrations are caused by vibrators and various power sources. For instance, a self-sustained oscillation caused by placing a plate into a flow whose critical velocity was overpassed (socalled fluttering) is a well-known phenomenon. To utilize such a fluttering phenomenon for energy-harvesting, the plate on which the piezoelectric elements were arranged was used, and the effect of their arrangement along the flow axis was considered. Then an optimization of the arrangement was performed among some positions and dimensions of piezoelectric elements [3].

The authors have also been interested in a mechanicalacoustic coupling problem. The representative example was 
TABLE 1: Mechanical and electrical properties of plate and piezoelectric element.

\begin{tabular}{lccc}
\hline & Density $\left[\mathrm{kg} / \mathrm{m}^{3}\right]$ & Young's modulus $[\mathrm{GPa}]$ & Poisson's ratio \\
\hline Aluminum plate & 2680 & 70.6 & 0.33 \\
\hline Electrode plate & 6900 & 100 & 0.35 \\
\hline Piezoelectric material & 8400 & 132 & 0.30 \\
& & $\gamma^{\varepsilon}=2.213 \times 10^{-9}[\mathrm{~F} / \mathrm{m}], d_{31}=-3.7 \times 10^{-12}[\mathrm{~m} / \mathrm{V}]$ & 0.6 \\
\hline
\end{tabular}

investigated as an architectural acoustic problem via a coupled panel-cavity system consisting of a rectangular box with slightly absorbing walls and a simply supported panel. The effect of the panel characteristics on the decay behavior of the sound field in the cavity was considered both theoretically and experimentally $[4,5]$. In an attempt to control noise in an airplane, an analytical model for investigating the coupling between the sound field in an aircraft cabin and the vibrations of the rear pressure bulkhead was proposed [6, 7]. A cylindrical structure adopted as the analytical model, in which the rear pressure bulkhead at one end of the cylinder was assumed to be a circular plate, was examined under various conditions. The plate was supported at its edges by springs whose stiffness could be adjusted to simulate the various support conditions. These investigations clarified the influence of the support conditions on the sound pressure of an internal sound field coupled with the vibration of the end plate. We used the above-mentioned analytical model $[6,7]$ of a cylindrical structure with plates at both ends to investigate the effect of the excitation frequency, at which the coupling system becomes nonperiodic owing to the application of excitation forces of different frequencies to the respective end plates, on the mechanical-acoustic coupling [8]. Then the effect of the excitation position with respect to the nodal lines on the appearance of vibration modes on the plates was investigated [9]. Finally, because a phase difference between both plate vibrations affects strongly mechanicalacoustic coupling, this coupling was considered in the cases, in which one end plate and both end plates were excited by a harmonic point force, respectively $[10,11]$. In particular, [10] made reference to the electricity generation with piezoelectric elements using coupling, as well as this paper. However, the effect of coupling was not clarified and has remained unclear, because the theoretical consideration was not carried out and the effect was not compared between the electricity generations using and not using coupling.

On the other hand, to suppress the above vibration and acoustic energy that were amplified by mechanical-acoustic coupling, an analytical model that included the installation of passive devices on the vibration system was proposed as the electromechanical-acoustic system. The effect was fully validated in the numerical approach owing to the damping technique using an RL shunt circuit, in which the piezoelectric device was incorporated, to the frequency characteristics of the coupling system [12]. Furthermore, this work was developed by adopting a more complex coupling system that comprised the plate equipped with shunted piezoelectric elements and interior and exterior acoustic fields coupled with plate vibration. This system was analyzed by means of a coupled finite element/boundary element method to control the noise radiation and sound transmission of the vibrating plate by passive piezoelectric techniques [13].

To develop a new electricity generation system, we adopt an analytical model similar to the above-mentioned cylindrical structure with plates at both ends, because the vibration area of the model on which piezoelectric elements can be installed is twice as large as that in the case of a single plate. The cylinder length is varied over a wide range while changing the plate thickness, while the harmonic point force is applied to one end plate and its frequency is selected to cause the plate to vibrate in the fundamental mode. The plate vibration induces electricity generation via electromechanical coupling with the piezoelectric effect of the surface-mounted piezoelectric element, while the plate vibration of the excitation side oscillates the other plate via mechanical-acoustic coupling. Consequently, the electromechanical-acoustic coupling problem must be considered and is estimated theoretically and experimentally from the electric power caused by the electricity generation, the mechanical power supplied to the plate, and the electricity generation efficiency that is derived from the ratio of both powers. In particular, by focusing on the electricity generation efficiency as the most significant characteristic, we verify that the performance of the proposed system is improved by using mechanical-acoustic coupling in comparison with using only the plate vibration without coupling. These results can be applied to power generation floors of roads, bridges, and so on, which can ensure a space to cause coupling. Recently, the monitoring system to grasp road surface information and tire conditions is developed and the vibration power generation is adopted as its power source. Since a tire has an inside space, we expect that these results are also useful at such a system design.

\section{Analytical Method}

2.1. Analytical Model. The analytical model consists of a cavity with two circular end plates, as shown in Figure 1. The plates are supported by translational and rotational springs distributed at constant intervals and the support conditions are determined by their respective spring stiffness $T$ and stiffness $R$. The plates of radius $r_{c}$ and thickness $h_{c}$ have Young's modulus $E_{c}$ and Poisson's ratio $v_{c}$. On the surfaces of both plates, piezoelectric elements are installed at the centers of the plates and have radius $r_{p}$, thickness $h_{p}$, Young's modulus $E_{p}$, and Poisson's ratio $v_{p}$. Then an electrode plate is sandwiched between the above plate and piezoelectric element and has radius $r_{b}$, thickness $h_{b}$, Young's modulus $E_{b}$, and Poisson's ratio $\nu_{b}$. The suffixes $c, p$, and $b$ herein indicate the circular plate, piezoelectric element, and 


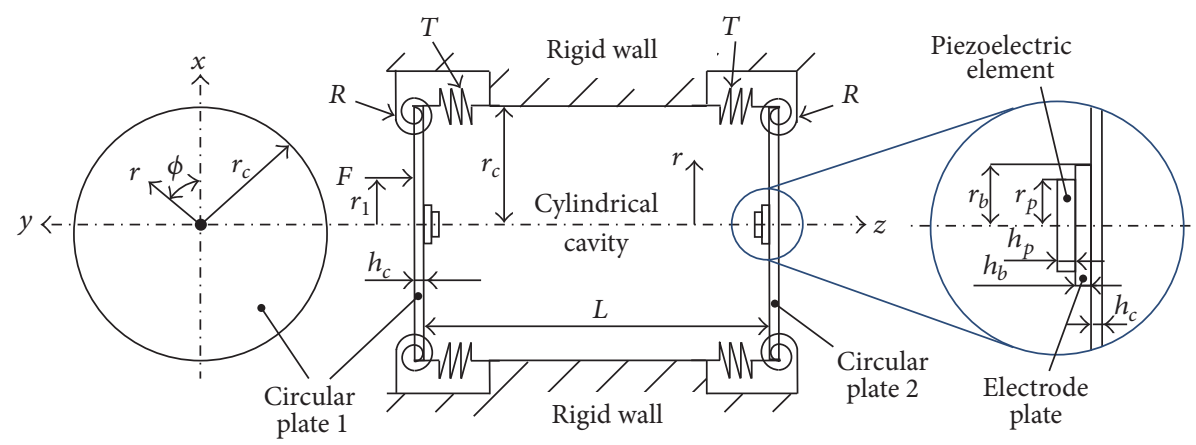

FIgURE 1: Configuration of analytical model.

electrode plate. On the other hand, the sound field, which is assumed to be cylindrical, has the same radius as that of the plates and varying length $L$ because the resonance frequency depends on the length. The boundary conditions are considered structurally and acoustically rigid at the lateral wall between the structure and sound field. The coordinates used are radius $r$, angle $\phi$ between the planes of the plates and the cross-sectional plane of the cavity, and distance $z$ along the cylinder axis. The periodic point force $F$ is applied to plate 1 at distance $r_{1}$ and angle $\phi_{1}$. The natural frequency of the plates is employed as the excitation frequency.

To formulate the plate motion, Hamilton's principle is applied to the analytical model:

$\delta H$

$$
\begin{aligned}
& =\delta \int_{t_{0}}^{t_{1}}\left(T_{c}+T_{p}+T_{b}-U_{c}-U_{p}-U_{b}-U_{s}+W\right) \mathrm{d} t \\
& =0
\end{aligned}
$$

where $H$ is the Hamiltonian; $T_{c}, T_{p}$, and $T_{b}$ are the kinetic energy of the circular plates, piezoelectric elements and electrode plates, respectively; and $U_{c}, U_{p}$, and $U_{b}$ are their respective potential energies. $U_{s}$ is the elastic energy stored in the springs, and $W$ is the total work done on plate 1 by the point force and on both plates by the sound pressure inside the cavity. Finally, $t_{0}$ and $t_{1}$ are two arbitrary times.

$w_{c 1}$ and $w_{c 2}$ are the flexural displacements of plates 1 and 2 , and $w_{p 1}$ and $w_{p 2}$ are those of the piezoelectric elements installed on the plates. Then suffixes 1 and 2 indicate plates 1 and 2 , respectively. They are found by substituting $X_{c n m}^{s}$ of (3) for the plate modes into (2) as suitable trial functions. The flexural displacements of the piezoelectric elements are identical to those of the plates, respectively, because it is assumed that the piezoelectric elements adhere completely to each circular plate through the electrode plate.

$$
\begin{aligned}
& w_{c 1}=w_{p 1}=\sum_{s=0}^{1} \sum_{n=0}^{\infty} \sum_{m=0}^{\infty} X_{n m}^{s} A_{1 n m}^{s} \mathrm{e}^{j\left(\omega t+\alpha_{1}\right)}, \\
& w_{c 2}=w_{p 2}=\sum_{s=0}^{1} \sum_{n=0}^{\infty} \sum_{m=0}^{\infty} X_{n m}^{s} A_{2 n m}^{s} \mathrm{e}^{j\left(\omega t+\alpha_{2}\right)},
\end{aligned}
$$

$$
X_{n m}^{s}=\sin \left(n \phi+\frac{s \pi}{2}\right)\left(\frac{r}{r_{c}}\right)^{m}
$$

where $n, m$, and $s$ are, respectively, the circumferential order, radial order, and symmetry index with respect to the plate vibration. $A_{1 \mathrm{~nm}}^{s}$ and $A_{2 \mathrm{~nm}}^{s}$ are coefficients to be determined, $\omega$ is the angular frequency of the harmonic point force acting on the plate, and $t$ is the elapsed time. $\alpha_{1}$ and $\alpha_{2}$ are the phases of the respective plate vibrations. In this analysis, $\alpha_{1}$ is set to $0^{\circ}$, and $\alpha_{2}$ ranges from $0^{\circ}$ to $180^{\circ}$.

2.2. Modelling of Piezoelectric Part. Only the piezoelectric part of plate 1 is used to explain its modelling in this section. The relationships of stress $\sigma_{p 1}$, strain $\varepsilon_{p 1}$, electric displacement $D_{1}$, and electric field $E_{1}$ are as follows:

$$
\left\{\begin{array}{c}
\sigma_{p 1} \\
D_{1}
\end{array}\right\}=\left[\begin{array}{cc}
E_{p}^{E} & -e^{T} \\
e & \gamma^{\varepsilon}
\end{array}\right]\left\{\begin{array}{c}
\varepsilon_{p 1} \\
E_{1}
\end{array}\right\} .
$$

$E_{p}^{E}$ signifies Young's modulus that was measured at a constant electric field, and $\gamma^{\varepsilon}$ indicates the dielectric constant that was measured at a constant strain. The above equation expresses relationships between electrical and mechanical characteristics of a piezoelectric element, and the stress is concretely related to the electric field by the piezoelectric coupling coefficient $e$. The piezoelectric coupling coefficient is expressed as

$$
e=d_{31} E_{p}^{E}
$$

where $d_{31}$ is the piezoelectric strain constant, in which the electric field occurs in the normal direction of the in-plane strain.

If the first variation is carried out in terms of the piezoelectric part on plate 1 , we can obtain

$$
\begin{gathered}
\delta \int_{t_{0}}^{t_{1}}\left(T_{p}-U_{p}\right) \mathrm{d} t=\frac{1}{2} \int_{t_{0}}^{t_{1}} \int_{V_{p 1}}\left\{\rho_{p} \dot{w}_{p 1} \delta \dot{w}_{p 1}\right. \\
\quad-\left(E_{p}^{E} \varepsilon_{p 1}-e E_{1}\right) \delta \varepsilon_{p 1} \\
\left.\quad+\left(e \varepsilon_{p 1}+\gamma^{\varepsilon} E_{1}\right) \delta E_{1}\right\} \mathrm{d} V_{p 1} \mathrm{~d} t,
\end{gathered}
$$


where $V_{p 1}$ is the volume of the piezoelectric element on plate 1. Here, the strain and electric charge are assumed as follows:

$$
\begin{aligned}
\varepsilon_{p 1} & =-z\left\{\frac{\partial^{2} w_{p 1}}{\partial r^{2}}+v\left(\frac{1}{r} \frac{\partial w_{p 1}}{\partial r}+\frac{1}{r^{2}} \frac{\partial^{2} w_{p 1}}{\partial \phi^{2}}\right)\right\} \\
& =-z X_{n m}^{s \prime \prime} A_{1 n m}^{s} \mathrm{e}^{j\left(\omega t+\alpha_{1}\right)}, \\
q_{1} & =B_{1 n m}^{s} \mathrm{e}^{j\left(\omega t+\alpha_{1}\right)} .
\end{aligned}
$$

$B_{1 n m}^{\mathcal{S}}$ is coefficient to be determined in this analysis as well as $A_{1 \mathrm{~nm}}^{s}$. Then the electric field $E_{1}$ is expressed by using its electric charge:

$$
\begin{aligned}
& E_{1}= Y_{n m} v_{1}=-R_{p} \dot{q}_{1}, \\
& Y_{n m}= \begin{cases}-\frac{1}{h_{p}} & \frac{h_{c}}{2}<z<\frac{h_{c}}{2}+h_{b}+h_{p}, \\
0 & -\frac{h_{c}}{2}<z<\frac{h_{c}}{2}+h_{b} .\end{cases}
\end{aligned}
$$

In this case, $v_{1}$ is the voltage that occurs in the electric field. We assume that the electric potential across the piezoelectric element is constant, since it is in the field that is not applied to the plate. Thus, $Y_{n m}$ is defined as described above. $R_{p}$ is the overall resistance value in an electricity generation circuit.

In order to easily express the electromechanical equation that is induced from (6), the elements $M_{p 1 n m m^{\prime}}^{s}$ and $K_{p 1 n m m^{\prime}}^{s}$ of the mass and stiffness matrices can be denoted as

$$
\begin{aligned}
M_{p 1 n m m^{\prime}}^{s} & =\int_{V_{p 1}} \rho_{p} X_{n m}^{s} X_{n m^{\prime}}^{s} \mathrm{~d} V_{p 1}, \\
K_{p 1 n m m^{\prime}}^{s} & =\int_{V_{p 1}} z^{2} X_{n m}^{s \prime \prime} E_{p}^{E} X_{n m^{\prime}}^{s \prime \prime} \mathrm{d} V_{p 1} .
\end{aligned}
$$

The index $m^{\prime}$ is also of a radial order and has a transposed relation to $m$. The elements $\theta_{1}$ and $C_{p 1}$ of the electromechanical coupling and capacitance matrices are defined as

$$
\begin{aligned}
\theta_{1} & =-\int_{V_{p 1}} z \rho_{p} X_{n m}^{s} e Y_{n m^{\prime}} \mathrm{d} V_{p 1}, \\
C_{p 1} & =\int_{V_{p 1}} Y_{n m} \gamma^{\varepsilon} Y_{n m^{\prime}} \mathrm{d} V_{p 1} .
\end{aligned}
$$

\subsection{Governing Equations of Electromechanical Coupling.} Here, the governing equation of electromechanical coupling with respect to plate 1 is determined. This equation defines the mechanical motion and electrical characteristics of this coupling system. The sound field inside the cavity or the vibration of plate 2 is not taken into consideration. The equation is obtained by applying the flexural displacement $w_{c 1}$ of (2) to the Hamiltonian $H$ of (1) in the same manner as (6) of the piezoelectric part that was derived in the previous section. Then the motion of plate 1 having a piezoelectric part and the electricity generation behavior of its piezoelectric element are governed by the following, respectively:

$$
\begin{aligned}
& \sum_{m^{\prime}=0}^{\infty}\left[\left\{K_{c 1 n m m^{\prime}}^{s}\left(1+j \eta_{c}\right)+K_{p 1 n m m^{\prime}}^{s}\left(1+j \eta_{p}\right)\right.\right. \\
& +K_{b 1 n m m^{\prime}}^{s}\left(1+j \eta_{b}\right) \\
& \left.\quad-\omega^{2}\left(M_{c 1 n m m^{\prime}}^{s}+M_{p 1 n m m^{\prime}}^{s}+M_{b 1 n m m^{\prime}}^{s}\right)\right\} \\
& \left.+r_{c} F_{s n}\left\{T_{1}+\left(\frac{m}{r_{c}}\right)\left(\frac{m^{\prime}}{r_{c}}\right) R_{1}\right\}\right] A_{1 n m^{\prime}}^{s} \mathrm{e}^{j\left(\omega t+\alpha_{1}\right)} \\
& \quad-\sum_{m^{\prime}=0}^{\infty} \theta_{1} v B_{1 n m^{\prime}}^{s} \mathrm{e}^{j\left(\omega t+\alpha_{1}\right)}=\mathbf{F}_{n m}^{s} \mathrm{e}^{j\left(\omega t+\alpha_{1}\right)}, \\
& \sum_{m^{\prime}=0}^{\infty} C_{p 1}^{-1} \theta_{1} A_{1 n m^{\prime}}^{s} \mathrm{e}^{j\left(\omega t+\alpha_{1}\right)}=\sum_{m^{\prime}=0}^{\infty}\left(j \omega R_{p}+C_{p 1}^{-1}\right) \\
& \cdot B_{1 n m^{\prime}}^{s} \mathrm{e}^{j\left(\omega t+\alpha_{1}\right)} .
\end{aligned}
$$

In (13), $K_{c 1 n m m^{\prime}}^{s}, K_{b 1 n m m^{\prime}}^{s}$ and $M_{c 1 n m m^{\prime}}^{s}, M_{b 1 n m m^{\prime}}^{s}$ are elements of the symmetrical stiffness and mass matrices with respect to the circular and electrode plates, respectively, because the index $m^{\prime}$ has a transposed relation to $m$, as well as $M_{p 1 n m m^{\prime}}^{s}$ and $K_{p 1 n m m^{\prime}}^{s} . \eta_{c}, \eta_{p}$, and $\eta_{b}$ are the structural damping factors of the circular plate, piezoelectric element, and electrode plate, respectively. Moreover, $F_{s n}$ is a coefficient that is determined by the indices $n$ and $s$, and $\mathbf{F}_{n m}^{s}$ is a load vector that expresses the point force. The details of $F_{s n}$ and the element $F_{n m}^{s}$ of the load vector are as follows:

$$
\begin{aligned}
& F_{s n}= \begin{cases}\pi, & \text { at } n \neq 0, \\
0, & \text { at } n=0, s=0, \\
2 \pi, & \text { at } n=0, s=1,\end{cases} \\
& F_{n m}^{s}=\int_{A_{1}} F \delta\left(r-r_{1}\right) \delta\left(\phi-\phi_{1}\right) X_{n m}^{s} \mathrm{~d} A_{1} .
\end{aligned}
$$

Here, $\delta$ is the delta function associated with the point force on plate 1 , whose area is denoted by $A_{1}$.

Since $A_{1 \mathrm{~nm}}^{s}$ and $B_{1 \mathrm{~nm}}^{s}$ can be obtained simultaneously from (13) and (14), the behavior of the plate vibration and the electricity generation under electromechanical coupling can be determined from (2) and (8). However, only the vibration of plate 1 is an analysis object and the behavior is assumed to be harmonic, so that $\exp \left\{j\left(\omega t+\alpha_{1}\right)\right\}$ can be eliminated in (13) and (14).

\subsection{Governing Equations of Electromechanical-Acoustic Cou-} pling. In this section, electromechanical-acoustic coupling is determined by adding a sound field inside the cavity to the electromechanical coupling introduced in the previous section. Naturally, the vibration of plate 2 is also added on the assumption that this coupling is phenomena based on the analytical model in Figure 1. The variation of (1) is carried out with respect to both plates. Consequently, the extremum 
of the Hamiltonian yields Euler's equations, which are the equations of motion of the respective plates.

$$
\begin{aligned}
& \sum_{m^{\prime}=0}^{\infty}\left[\left\{K_{c 1 n m m^{\prime}}^{s}\left(1+j \eta_{c}\right)+K_{p 1 n m m^{\prime}}^{s}\left(1+j \eta_{p}\right)\right.\right. \\
& +K_{b 1 n m m^{\prime}}^{s}\left(1+j \eta_{b}\right) \\
& \left.\quad-\omega^{2}\left(M_{c 1 n m m^{\prime}}^{s}+M_{p 1 n m m^{\prime}}^{s}+M_{b 1 n m m^{\prime}}^{s}\right)\right\} \\
& \left.\quad+r_{c} F_{s n}\left\{T_{1}+\left(\frac{m}{r_{c}}\right)\left(\frac{m^{\prime}}{r_{c}}\right) R_{1}\right\}\right] A_{1 n m^{\prime}}^{s} \mathrm{e}^{j \alpha_{1}} \\
& \quad-\sum_{m^{\prime}=0}^{\infty} \theta_{1} v B_{1 n m^{\prime}}^{s} \mathrm{e}^{j \alpha_{1}}=\mathbf{F}_{n m}^{s} \mathrm{e}^{j \alpha_{1}}-\mathbf{P}_{1 n m^{s}}^{s} \\
& \sum_{m^{\prime}=0}^{\infty}\left[\left\{K_{c 2 n m m^{\prime}}^{s}\left(1+j \eta_{c}\right)+K_{p 2 n m m^{\prime}}^{s}\left(1+j \eta_{p}\right)\right.\right. \\
& \quad+K_{b 2 n m m^{\prime}}^{s}\left(1+j \eta_{b}\right) \\
& \left.\quad-\omega^{2}\left(M_{c 2 n m m^{\prime}}^{s}+M_{p 2 n m m^{\prime}}^{s}+M_{b 2 n m m^{\prime}}^{s}\right)\right\} \\
& \left.\quad+r_{c} F_{s n}\left\{T_{2}+\left(\frac{m}{r_{c}}\right)\left(\frac{m^{\prime}}{r_{c}}\right) R_{2}\right\}\right] A_{2 n m^{\prime}}^{s} \mathrm{e}^{j \alpha_{2}} \\
& \quad-\sum_{m^{\prime}=0}^{\infty} \theta_{2} v B_{2 n m^{\prime}}^{s} \mathrm{e}^{j \alpha_{2}}=\mathbf{P}_{2 n m^{s}}^{s} .
\end{aligned}
$$

Equations (16) and (17) are obviously the motion equations of plates 1 and 2, respectively, from the subscripts of each element constructing both equations, since the elements correspond to them in (13). However, $\exp \left(j \alpha_{1}\right)$ and $\exp \left(j \alpha_{2}\right)$ remained in (16) and (17), respectively, since both vibrations have an effect on each other via mechanical-acoustic coupling. Equation (16) has the point force and acoustic excitation terms on the right-hand side, because plate 1 is simultaneously subjected to both excitations. However, the point force is not applied to plate 2, with the result that (17) consists of only the acoustic term, which also functions as the coupling term between each plate vibration and the sound field. The elements of the acoustic excitation vectors are expressed as

$$
\begin{aligned}
& P_{1 n m}^{s}=\int_{A_{1}} P_{s} X_{n m}^{s} \mathrm{~d} A_{1}, \\
& P_{2 n m}^{s}=\int_{A_{2}} P_{s} X_{n m}^{s} \mathrm{~d} A_{2} .
\end{aligned}
$$

Here, $P_{s}$ is the sound pressure at an arbitrary point on the boundary surface of the plates, and $A_{2}$ signifies the area of plate 2 .

This procedure also derives the equations representing electrical characteristics in this coupling system. They are as follows:

$$
\begin{aligned}
& \sum_{m^{\prime}=0}^{\infty} C_{p 1}^{-1} \theta_{1} A_{1 n m^{\prime}}^{s}=\sum_{m^{\prime}=0}^{\infty}\left(j \omega R_{p}+C_{p 1}^{-1}\right) B_{1 n m^{\prime}}^{s}, \\
& \sum_{m^{\prime}=0}^{\infty} C_{p 2}^{-1} \theta_{2} A_{2 n m^{\prime}}^{s}=\sum_{m^{\prime}=0}^{\infty}\left(j \omega R_{p}+C_{p 2}^{-1}\right) B_{2 n m^{\prime}}^{s} .
\end{aligned}
$$

The above elements constructing the respective equations correspond to those of (14), as well as (16) and (17).

In this investigation, the acoustic modal shape $Z_{n p q}^{s}$ and angular resonance frequency $\omega_{n p q}$ in the cavity (where the indices $n, p$, and $q$ indicate the circumferential, radial, and longitudinal orders, resp.) are defined as

$$
\begin{aligned}
& Z_{n p q}^{s}=\sin \left(n \phi+\frac{s \pi}{2}\right) J_{n}\left(\lambda_{n p} r\right) \cos \left\{\left(\frac{q \pi}{L}\right) z\right\}, \\
& \omega_{n p q}=c\left\{\lambda_{n p}{ }^{2}+\left(\frac{q \pi}{L}\right)^{2}\right\}^{1 / 2},
\end{aligned}
$$

where $J_{n}$ is the $n$ th-order Bessel function, $c$ is the cavity speed of sound, and $\lambda_{n p}$ is the $p$ th solution of an eigenvalue problem for a circular sound field having modes $(n, p)$ divided by the plate radius $r_{c}$. The boundary conditions between the plate vibrations and sound field on the respective plate surfaces are found by assuming continuity of the velocities on the plates:

$$
\begin{aligned}
& \left(\frac{\partial P_{s}}{\partial \mathbf{u}}\right)_{z=0}=\rho_{s} \omega^{2} w_{c 1}, \\
& \left(\frac{\partial P_{s}}{\partial \mathbf{u}}\right)_{z=L}=-\rho_{s} \omega^{2} w_{c 2},
\end{aligned}
$$

where $P_{s}$ is the sound pressure within and on the surface bounding the medium and $\mathbf{u}$ is the unit normal to the boundary surface (positive towards the outside). However, $\partial P s / \partial \mathbf{u}$ is 0 on the lateral wall of the cylinder since the wall remains rigid.

Because the analytical model has two boundary surfaces, we can apply Green's function $G$ to (21), so that the sound pressure $P_{s}$ inside the cavity becomes

$$
P_{s}=-\int_{A_{1}} G \rho_{s} \omega^{2} w_{c 1} \mathrm{~d} A_{1}+\int_{A_{2}} G \rho_{s} \omega^{2} w_{c 2} \mathrm{~d} A_{2}
$$

In addition, $P_{s}$ can also be expressed as follows $[6,7]$ :

$$
P_{s}=\rho_{s} c^{2} \sum_{p=1}^{\infty} \sum_{q=0}^{\infty} \frac{P_{n p q}^{s} Z_{n p q}^{s}}{M_{n p q}^{s}}
$$

where $\rho_{s}$ is the fluid density in the cavity, $P_{n p q}^{s}$ is the pressure coefficient to be determined, and $M_{n p q}^{s}$ is the mean value of $Z_{n p q}^{s}{ }^{2}$ averaged over the sound field.

The equation relating (22) and (23) is obtained by applying Green's function [14] to an arbitrary acoustic mode $(n, p, q)$ as

$$
\begin{aligned}
& \left(\omega_{n p q}^{2}-\omega^{2}\right) P_{n p q}^{s} \\
& \quad=-\frac{\omega^{2}}{V_{s}}\left(\int_{A_{1}} Z_{n p q}^{s} w_{c 1} \mathrm{~d} A_{1}+\int_{A_{2}} Z_{n p q}^{s} w_{c 2} \mathrm{~d} A_{2}\right) .
\end{aligned}
$$


Substituting (2) for $w_{c 1}$ and $w_{c 2}$ and considering a modal damping factor $\eta_{s}$, (24) can be rewritten as

$$
\begin{aligned}
& \left(\omega_{n p q}^{2}+j \eta_{s} \omega_{n p q} \omega-\omega^{2}\right) P_{n p q}^{s} \\
& =\frac{A \omega^{2}}{V_{s}}\left(-\sum_{m=0}^{\infty} I_{1} A_{1 n m}^{s} \mathrm{e}^{j \alpha_{1}}+\sum_{m=0}^{\infty} I_{2} A_{2 n m}^{s} \mathrm{e}^{j \alpha_{2}}\right), \\
& I_{1}=\frac{1}{A} \int_{A_{1}} X_{n m}^{s} Z_{n p q}^{s} \mathrm{~d} A_{1} \\
& I_{2}=\frac{1}{A} \int_{A_{2}} X_{n m}^{s} Z_{n p q}^{s} \mathrm{~d} A_{2},
\end{aligned}
$$

where $A$ is the total surface area of the plates, $V_{s}$ is the cavity volume, and then $I_{1}$ and $I_{2}$ are the spatial coupling coefficients. Moreover, substituting (23) for $P_{s}$ and applying $I_{1}$ and $I_{2}$ to the integrals in (18), the acoustic excitation terms $P_{1 n m}^{s}$ and $P_{2 n m}^{s}$ can be expressed with respect to an arbitrary vibration mode $(n, m)$ as

$$
\begin{aligned}
& P_{1 n m}^{s}=\rho_{s} c^{2} A \sum_{p=1}^{\infty} \sum_{q=0}^{\infty} \frac{I_{1} P_{n p q}^{s}}{M_{n p q}^{s}}, \\
& P_{2 n m}^{s}=\rho_{s} c^{2} A \sum_{p=1}^{\infty} \sum_{q=0}^{\infty} \frac{I_{2} P_{n p q}^{s}}{M_{n p q}^{s}} .
\end{aligned}
$$

Finally, replacing $P_{n p q}^{s}$ in (27) with those in (25) and then inserting them in (16) and (17), we can complete the electromechanical-acoustic coupling equations in conjunction with (19). The details on the respective right-hand sides of (16) and (17) are

$$
\begin{gathered}
\mathbf{F}_{n m}^{s} \mathrm{e}^{j \alpha_{1}}-\mathbf{P}_{1 n m}^{s}=\mathbf{F}_{n m}^{s} \mathrm{e}^{j \alpha_{1}}+\frac{\rho_{s} c^{2} \omega^{2} A^{2}}{V_{s}} \\
\cdot \sum_{m^{\prime}=0}^{\infty} \sum_{p=1}^{\infty} \sum_{q=0}^{\infty} \frac{I_{1}\left(I_{1} A_{1 n m^{\prime}}^{s} \mathrm{e}^{j \alpha_{1}}-I_{2} A_{2 n m^{\prime}}^{s} \mathrm{e}^{j \alpha_{2}}\right)}{M_{n p q}^{s}\left(\omega_{n p q}^{2}+j \eta_{s} \omega_{n p q} \omega-\omega^{2}\right)}, \\
\mathbf{P}_{2 n m}^{s}=\frac{\rho_{s} c^{2} \omega^{2} A^{2}}{V_{s}} \\
\cdot \sum_{m^{\prime}=0}^{\infty} \sum_{p=1}^{\infty} \sum_{q=0}^{\infty} \frac{I_{2}\left(I_{1} A_{1 n m^{\prime}}^{s} \mathrm{e}^{j \alpha_{1}}-I_{2} A_{2 n m^{\prime}}^{s} \mathrm{e}^{j \alpha_{2}}\right)}{M_{n p q}^{s}\left(\omega_{n p q}^{2}+j \eta_{s} \omega_{n p q} \omega-\omega^{2}\right)} .
\end{gathered}
$$

On the right-hand sides, $\mathbf{P}_{1 n m}^{s}$ and $\mathbf{P}_{2 n m}^{s}$ show the acoustic excitation for plates 1 and 2 , respectively. The acoustic excitation terms have both $I_{1}$ and $I_{2}$ since the acoustic mode of the sound field is coupled with the vibration modes of the respective plates. In actual calculation, the relationships between $A_{1 n m}^{s}$ and $B_{1 n m}^{s}$ and between $A_{2 n m}^{s}$ and $B_{2 n m}^{s}$ are obtained from (19) and are applied to (16) and (17), respectively, with the result that $A_{1 n m}^{s}, A_{2 n m}^{s}, B_{1 n m}^{s}$, and $B_{2 n m}^{s}$ can be derived by solving the above simultaneous equations (16) and (17).

The flexural displacements $w_{c 1}$ and $w_{c 2}$ of plates 1 and 2 are obtained from (2) by employing $A_{1 \mathrm{~nm}}^{s}$ and $A_{2 \mathrm{~nm}}^{s}$ determined above. The voltage $v_{1}$ based on the vibration of plate 1 is derived from (8) and (9) by employing the above $B_{1 n m}^{s}$. Then the voltage $v_{2}$ based on the vibration of plate 2 is also obtained in the same manner by employing the above $B_{2 n m}^{s}$. However, the procedure to calculate $P_{s}$ involves substituting $P_{n p q}^{s}$ in (25) for that in (23). Solved from (29), into which $P_{s}$ is inserted, over the entire volume of the cavity, the sound pressure $P_{v}$ is estimated by the logarithmic value $L_{p v}$ relative to $P_{0}=4 \times 10^{-10} \mathrm{~N}^{2} / \mathrm{m}^{4}$, as shown in (30) as follows:

$$
\begin{aligned}
P_{v} & =\frac{1}{2 V_{s}} \int_{V_{s}} P_{s} P_{s}^{*} \mathrm{~d} V_{s}, \\
L_{p v} & =10 \log \frac{P_{v}}{P_{0}},
\end{aligned}
$$

where $P_{c}^{*}$ is the conjugate component.

\section{Experimental Apparatus and Method}

Figure 2 shows the configuration of the experimental apparatus used in this study. The structure consists of a steel cylinder with circular aluminum end plates whose thickness $h_{c}$ varies at 2.0,2.5, 3.0, and $4.0 \mathrm{~mm}$. The cylinder has an inner radius of $153 \mathrm{~mm}$, and the length $L$ can range from 500 to $2000 \mathrm{~mm}$ to emulate the analytical model. Plate 1 is subjected to the point force, whose frequency makes the plate excite in the $(0,0)$ mode; and amplitude $F$ is controlled to be $1 \mathrm{~N}$, excited by a small vibrator. The position of the point force $r_{1}$ is normalized by radius $r_{c}$ and is set to $r_{1} / r_{c}=0.4$.

In the excitation experiment, to estimate the mechanical power $P_{m}$ supplied to plate 1 by the small vibrator, an acceleration sensor is installed near the position of the point force on plate 1 , and $P_{m}$ is predicted from the point force and acceleration $a_{1}$. The phase difference between the plate vibrations is also measured owing to the installation of the acceleration sensor at the same position on plate 2 , resulting in significant effects on the mechanical-acoustic coupling. To estimate the internal acoustic characteristics, the sound pressure level in the cavity is measured using condenser microphones with a probe tube. The tips of the probe tubes are located near the plates and the cylinder wall, which are the approximate locations of the maximum sound pressure level when the sound field becomes resonant.

To perform the electricity generation experiment, the piezoelectric element is used. It is comprised of a piezoelectric part constructed of ceramics and an electrode part constructed of brass, which have radiuses $r_{p}$ and $r_{b}$ of 12.5 and $17.5 \mathrm{~mm}$ and thicknesses $h_{p}$ and $h_{b}$ of 0.23 and $0.30 \mathrm{~mm}$, respectively. The piezoelectric elements are installed at the centers of both plates. The electric powers $P_{e 1}$ and $P_{e 2}$ generated by the expansion and contraction of the piezoelectric elements on plates 1 and 2 are discharged through the resistance circuit, which consists of three resistors having resistances $R_{v}, R_{i}$, and $R_{c}$, as shown in Figure 2(b). $R_{v}$ and $R_{i}$ are the resistances of the voltmeter and ammeter, which are built into the wattmeter, and are $2 \mathrm{M} \Omega$ and $2 \mathrm{~m} \Omega$, respectively, while $R_{c}$ is the resistance of the resistor connected outside the wattmeter and is $97.5 \mathrm{k} \Omega$. To grasp the effect of mechanicalacoustic coupling on energy-harvesting, the electric power 


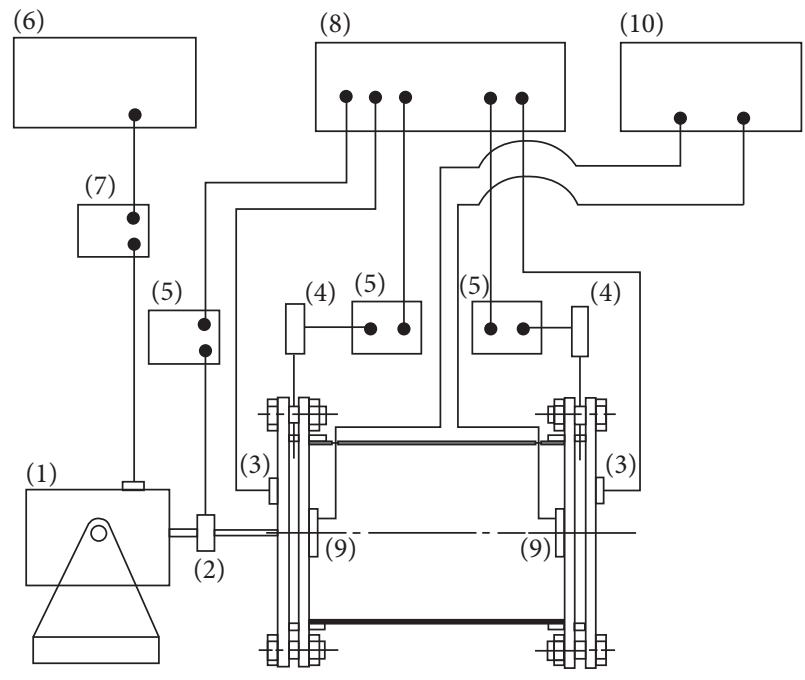

(a)

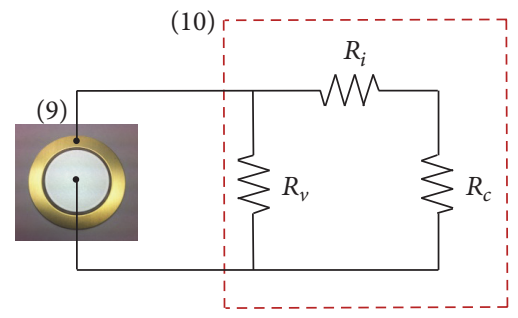

(b)

FIGURE 2: Configuration of experimental apparatus: (a) measurement system and (b) electrical circuit of energy-harvesting device. (1) Vibration generator, (2) load cell, (3) acceleration sensor, (4) condenser microphone, (5) amplifier, (6) multifunction generator, (7) power supply, (8) FFT analyzer, (9) piezoelectric element, and (10) power meter.

and other data are also measured without the cylinder (i.e., in the electricity generation under the vibration of only plate 1) and are estimated in comparison with those with cylinder. In such an estimation, electricity generation efficiency is used and is derived from the electric power normalized by the mechanical power $P_{m}$ supplied to plate 1 by the vibrator. However, the electricity generation efficiencies, which are obtained from $P_{e 1}, P_{e 2}$, and their total electric power $P_{e}$, are denoted by $P_{e m 1}, P_{e m 2}$, and $P_{e m}$, respectively, when electromechanical coupling is taken into consideration.

\section{Results and Discussion}

4.1. Electricity Generation Characteristics Based on Plate Vibration. The electricity generation caused by the piezoelectric element, which adheres to the center of plate 1 excited by the vibrator, is considered, so that the cavity and plate 2 are not assumed in the analytical model of Figure 1 and are actually eliminated in the experimental apparatus of Figure 2. The plate and piezoelectric element are the same as the experimental apparatus in the respective dimensions and have mechanical and electrical properties as shown in Table 1. However, only $h_{c}=3 \mathrm{~mm}$ is employed as the thickness of plate 1 in this section. The support condition of plate 1 , which has flexural rigidity $D\left[=E_{c} h_{c}^{3} /\left\{12\left(1-v_{c}^{2}\right)\right\}\right]$, is expressed by the nondimensional stiffness parameters $T_{n}\left(=T_{c} r_{c}^{3} / D\right)$ and $R_{n}\left(=R_{c} r_{c} / D\right)$. If $R_{n}$ ranges from 0 to $10^{8}$ when $T_{n}$ is $10^{8}$, the support condition can be assumed, from a simple support to a clamped support.

Figure 3 shows the theoretical and experimental accelerations $a_{1}$ at the excitation point as functions of the excitation frequency $f$. Before obtaining these results, we carried out experimental modal analysis and made sure of the natural frequency of plate 1 . Then the actual condition adopts $T_{n}=10^{8}$

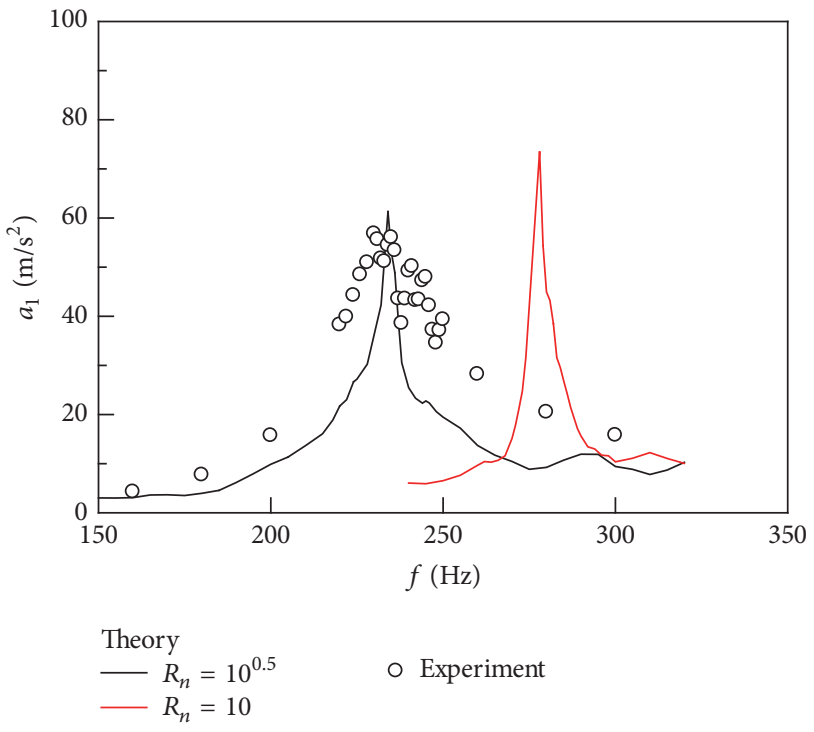

FIgURE 3: Acceleration at excitation point as function of excitation frequency.

and $R_{n}=10^{1}$ to get closer to the experimental support condition. Because this support condition brings the natural frequency to $280 \mathrm{~Hz}$, the corresponding $a_{1}$ reaches the peak at the natural frequency. However, the experimental $a_{1}$ peaks around $f=230 \mathrm{~Hz}$ and the actual natural frequency is shifted to a lower frequency region than the above natural frequency. Under a constraint of the experimental apparatus, the load cell cannot be directly installed on plate 1 , so that the point force is applied to plate 1 via a stick from the vibration generator. Therefore, an additional mass derived from the stick contributes to shift the natural frequency to the lower frequency region, not avoided in this experiment. 


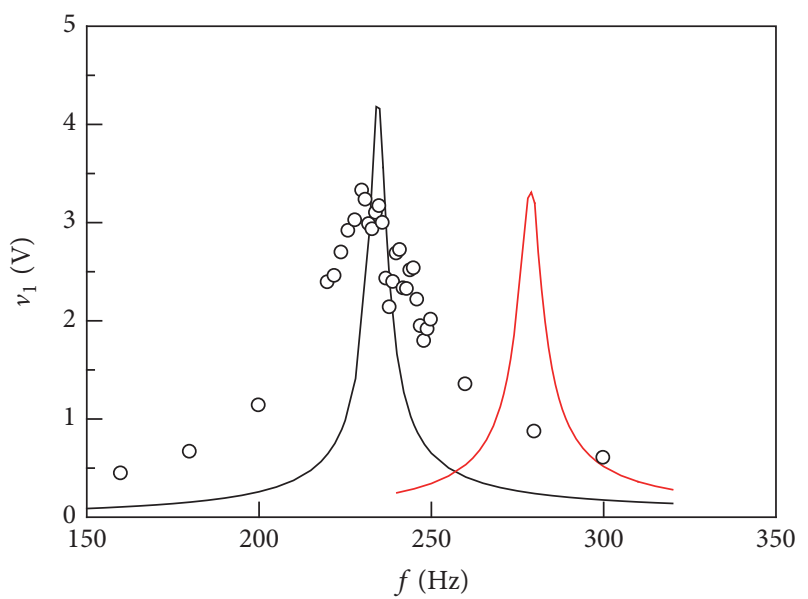

$\begin{array}{ll}\text { Theory } & \\ -R_{n} & =10^{0.5} \\ R_{n} & =10\end{array} \quad$ ○ Experiment

FIgURE 4: Voltage with electricity generation as function of excitation frequency.

Moreover, because the shift in the natural frequency cannot be explained only by such an additional mass, characteristics of the vibration generator are also considered as other factors. The flexural deflection of plate 1 excited by the vibration generator is suppressed by the generator characteristics and the excitation position in comparison with the free vibration. In general, a vibration system receiving damping force becomes lower in the natural frequency than a free vibration system. Because of those, we suppose that such a shift in the natural frequency is caused by some factors.

Since it is difficult to theoretically express the actual situation at the moment, a theoretical consideration is attempted by shifting the natural frequency of plate 1 to the experimental peak frequency owing to substituting $10^{0.5}$ for $R_{n}$. By dealing with such an attempt, its behavior is similar to the experimental $a_{1}$. Figure 4 shows the voltage $v_{1}$ with electricity generation as functions of $f$. The theoretical $v_{1}$ has peaks at $f=234$ and $278 \mathrm{~Hz}$ that are derived from $R_{n}=10^{0.5}$ and $10^{1}$, respectively, and the experimental $v_{1}$ is maximized around $f=230 \mathrm{~Hz}$. These correspond to $a_{1}$ very well.

In electricity generation by means of the plate vibration with piezoelectric elements, coupling between the structural vibration and electric field (i.e., electromechanical coupling) should be considered to relate the in-plane stress to the applied electric field. The electricity generation characteristics are considered by the electric power $P_{e 1}$ via the piezoelectric element and the mechanical power $P_{m}$ supplied to plate 1 by the vibrator. Figure 5 shows the relationship between $P_{e 1}$ and $P_{m}$ at $f=234$ and $280 \mathrm{~Hz}$ of $R_{b}=10^{0.5}$. The experimental results corresponding to those are also shown. In this figure, both axes are expressed logarithmically, because $P_{e 1}$ at $f=$ $280 \mathrm{~Hz}$ is considerably smaller than that of $f=234 \mathrm{~Hz}$ even if we can make sure of it from Figure 4 and is hardly exhibited together with that at $f=234 \mathrm{~Hz}$ on the ordinary axes. With respect to the relationship between $P_{e 1}$ and $P_{m}$, linear changes mean that $P_{e 1}$ is directly proportional to $P_{m}$,

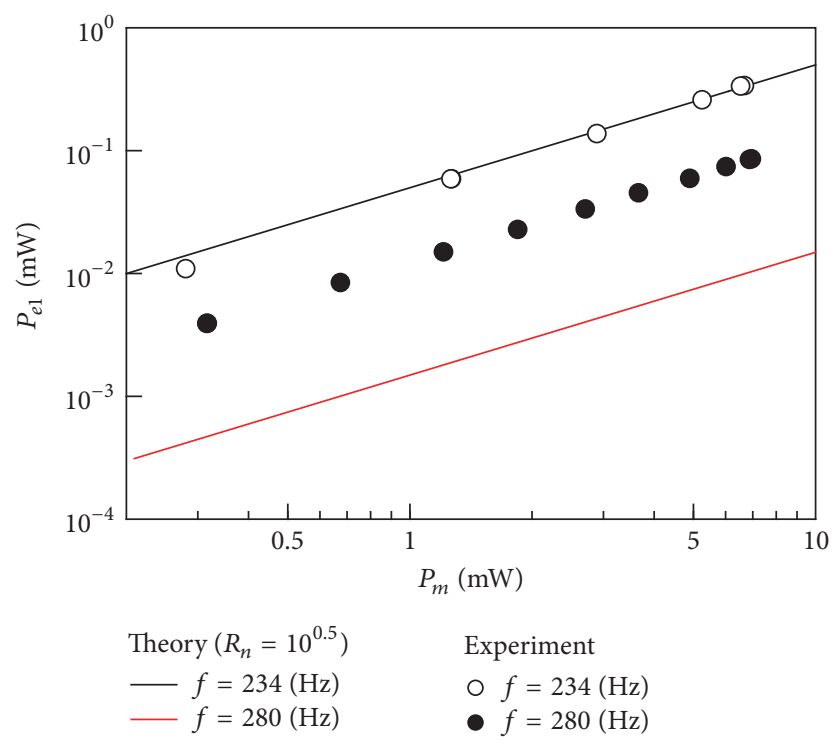

FIGURE 5: Relationship between electric power caused by electricity generation and mechanical power supplied from vibrator.

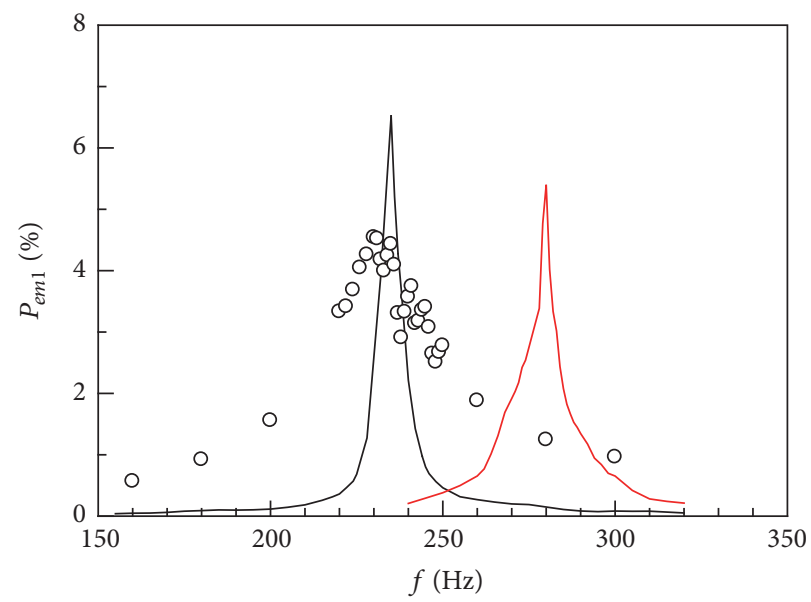

$$
\begin{aligned}
& \text { Theory } \\
& -R_{n}=10^{0.5} \\
& R_{n}=10
\end{aligned}
$$

FIGURE 6: Variations in electricity generation efficiency with excitation frequency.

and the difference of $P_{e 1}$ in the changes indicates that of the rate of changes in $P_{e 1}$ for $P_{m}$; that is, the difference is identical to that of inclinations of changes in $P_{e 1}$ for $P_{m}$ on the ordinary axes.

The theoretical and experimental $P_{e 1}$, which is derived from the peak voltages in Figure 4, increases similarly to $P_{m}$, while the theoretical $P_{e 1}$ at $f=280 \mathrm{~Hz}$ is suppressed in comparison with that at $f=234 \mathrm{~Hz}$, and the difference from the experimental $P_{e 1}$ at $f=280 \mathrm{~Hz}$ is expanded. The additional mass of the stick affects not only the natural frequency but also the mechanical power supplied to the plate 1 because a force derived from the additional mass is added to the point force, so that $P_{e 1}$ also increases in response to $P_{m}$. 
Since the effect is remarkable relatively at $f=280 \mathrm{~Hz}$ that is far away from the peak frequency, we consider that these experimental results are somewhat overestimated.

Figure 6 shows the electricity generation efficiency $P_{e m 1}$ as functions of $f$ and also the comparison between theoretical and experimental results in Figures 3 and 4 . The theoretical $P_{e m 1}$ of $R_{n}=10^{0.5}$ and $10^{1}$ peaks at $f=234$ and $280 \mathrm{~Hz}$, respectively, and decreases as the natural frequency increases. The experimental $P_{e m 1}$ varies similarly to the above $P_{e m 1}$ of $R_{n}=10^{0.5}$, with the result that it demonstrates the theoretical consideration and that such an electricity generation system does not function effectively in any frequency range except the natural frequency region.

4.2. Electricity Generation Characteristics Using MechanicalAcoustic Coupling. In this section, the cylinder and plate 2 are added to the above theoretical and experimental models which were introduced in the previous section; that is, they are models as shown in Figures 1 and 2(a). In the analytical model, the dimensions and so on of plate 2 are identical to those of plate 1 , the support condition adopts $T_{n}=10^{8}$ and $R_{n}=10^{1}$ in both plates, and the point force $F$ is set to $1 \mathrm{~N}$. The cylinder has the same radius as that of plates 1 and 2, and its length $L$ ranges from 100 to $2000 \mathrm{~mm}$.

Figure 7 shows the sound pressure level $L_{p v}$, which is averaged over the entire volume of the cavity and is maximized at each $L$ when $h_{c}$ and $f$ are set to $3 \mathrm{~mm}$ and $280 \mathrm{~Hz}$, respectively, and the phase $\alpha_{2}$ ranges from 0 to $180^{\circ}$ as functions of $L$. The theoretical level $L_{p v}$ peaks at 610, 1230 , and $1840 \mathrm{~mm}$. The peaks are caused by the promotion of mechanical-acoustic coupling between the plate vibration and acoustic modes. Then the acoustic modes are the $(0,0,1)$, $(0,0,2)$, and $(0,0,3)$ modes whose plane modal shape is similar to that of plate vibration mode $(0,0)$. To validate these theoretical results, the sound pressure levels $L_{p 1}$ and $L_{p 2}$, which are measured near plates 1 and 2, are also indicated. The experimental peaks occur around the lengths where $L_{p v}$ peaks, whereas $L_{p 1}$ decreases remarkably around $L=950$ and $1600 \mathrm{~mm}$ in the process of shifting acoustic modes because of changing $L$.

To consider the effect of mechanical-acoustic coupling on electricity generation characteristics, Figure 8 shows the experimental accelerations $a_{1}$ and $a_{2}$ of plates 1 and 2 as functions of $L$ under the above coupling. Since plate 1 is excited at $f=280 \mathrm{~Hz}$ via the stick connected with the vibrator, $a_{1}$ remains low and almost constant over the entire range of $L$, having small increases at $L=620,1250$, and $1880 \mathrm{~mm}$. These results can be also predicted from those in Figure 3. On the other hand, the cylindrical sound field connecting with plate 1 is formed via mechanical-acoustic coupling, and plate 2 is excited by the sound field without the point force. As a result, $a_{2}$ of plate 2 has specific peaks at $L=620,1250$, and $1880 \mathrm{~mm}$, suppressed in the other ranges of $L$, because the natural frequency of plate 2 becomes $280 \mathrm{~Hz}$. Figure 9 shows the voltages $v_{1}$ and $v_{2}$ based on the electricity generations of plates 1 and 2 as functions of $L . v_{1}$ and $v_{2}$ are directly proportional to $a_{1}$ and $a_{2}$, respectively, depending on the in-plane strain of the plate that is determined by the

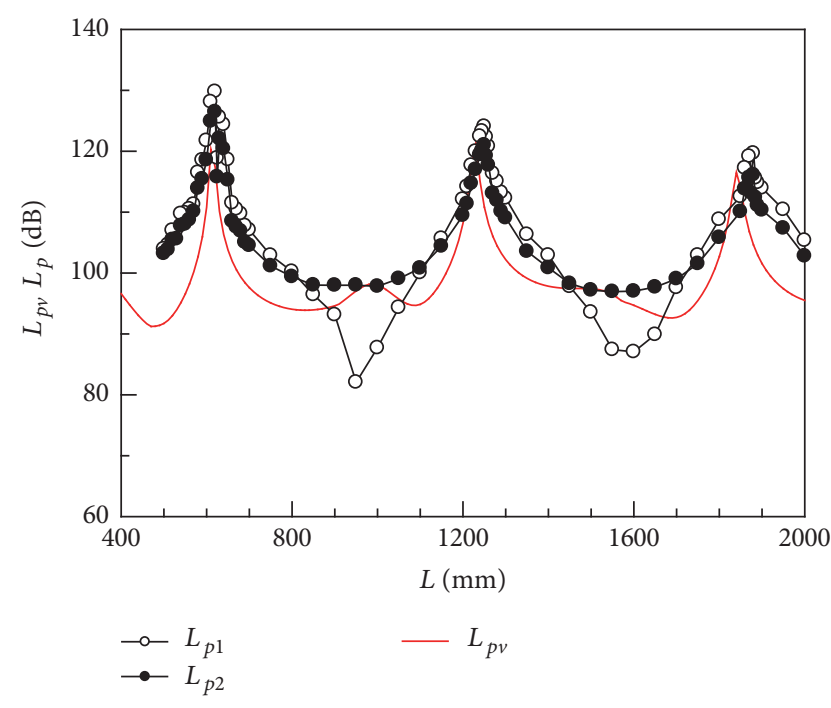

FIGURE 7: Sound pressure level inside cavity as function of cylinder length.

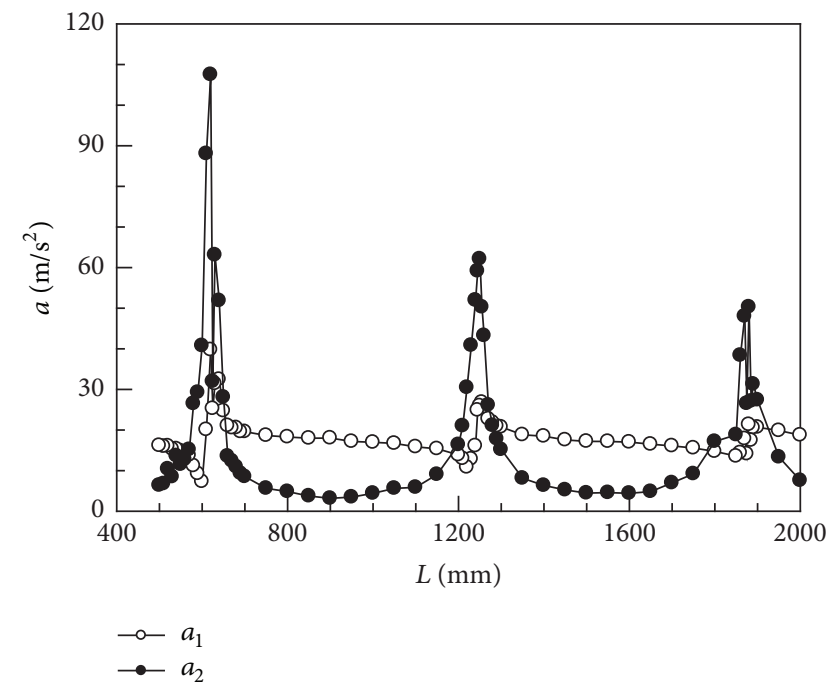

FIGURE 8: Acceleration at both plates as function of cylinder length.

out-of-plane deflection of the plate. Therefore, $v_{1}$ and $v_{2}$ have a tendency that is similar to that of $a_{1}$ and $a_{2}$; in particular, $v_{2}$ is much larger than $v_{1}$ at $L=620 \mathrm{~mm}$, at which coupling with the $(0,0,1)$ mode is promoted, as well as the behavior of $a_{1}$ and $a_{2}$.

The electricity generation efficiencies $P_{e m 1}, P_{e m 2}$, and $P_{e m}$ are calculated from the relationship between electric power via the piezoelectric element and mechanical power supplied to plate 1 . They are shown with changing $L$ in Figure 10 . They are derived from the above results so that $P_{e m 1}$ of plate 1 remains almost constant over the entire range of $L$, and $P_{e m 2}$ of plate 2 increases greatly at $L=620,1250$, and $1880 \mathrm{~mm}$. It is natural that $P_{e m}$ peaks at those lengths and remains almost constant in the other ranges of $L$, calculated from both electric power values of plates 1 and 2. Because 


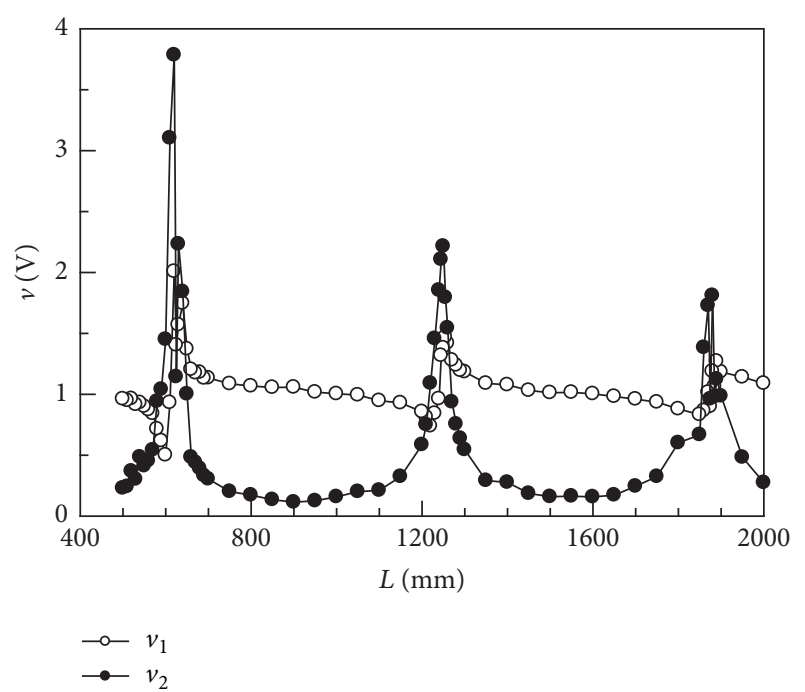

Figure 9: Voltages with respective electricity generations at both plates as function of cylinder length.

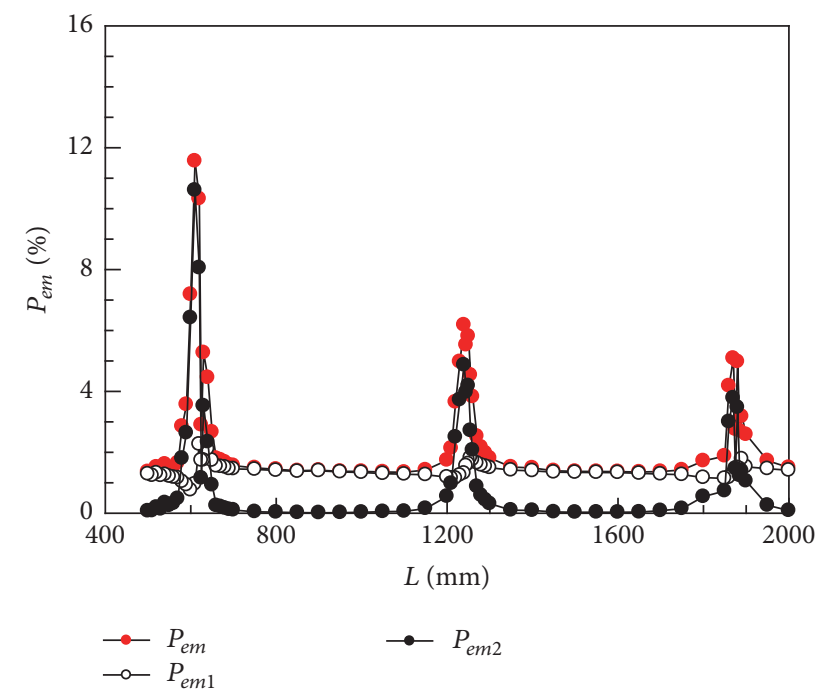

FIGURE 10: Variations in electricity generation efficiency with cylinder length.

the above characteristics of the electricity generation efficiency are affected by mechanical-acoustic coupling, whose characteristics are influenced by the relationship between the natural frequencies of the plate vibrations and internal sound field, the other thicknesses are also employed in this study. Figure 11 shows $P_{e m}$ of $h_{c}=2$ and $4 \mathrm{~mm}$, whose respective natural frequencies are 190 and $390 \mathrm{~Hz}$, as functions of $L . P_{e m}$ reaches the respective maximum values caused by coupling with the $(0,0,1)$ mode at $L=850$ and $455 \mathrm{~mm}$ when $h_{c}=2$ and $4 \mathrm{~mm}$. The promotion of mechanical-acoustic coupling has been confirmed by theoretical and experimental acoustic characteristics.

4.3. Variation in Electricity Generation Efficiency with Plate Thickness. As described in the previous section, the electricity generation characteristics are strongly affected by the shift

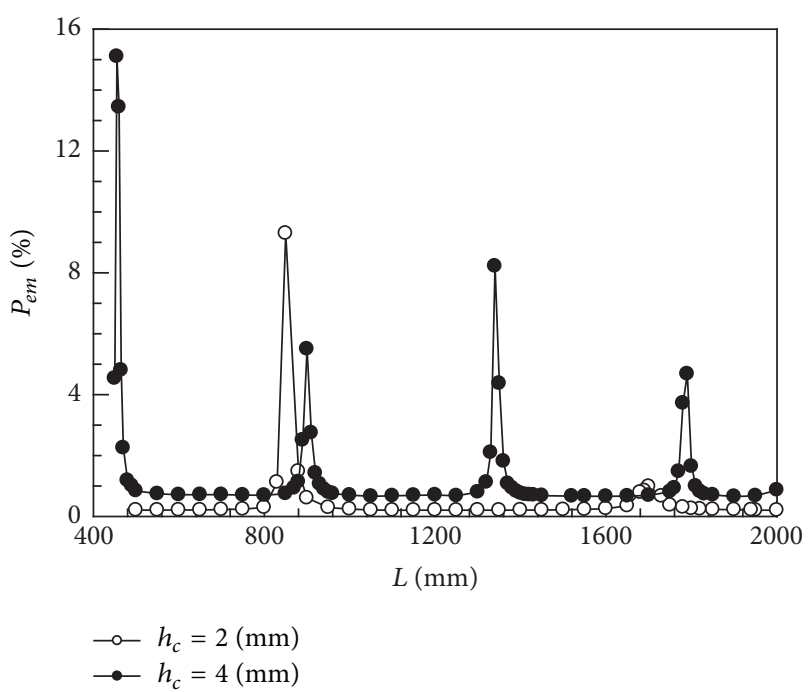

FIGURE 11: Comparison between electricity generation efficiencies when plate thicknesses are 2 and $4 \mathrm{~mm}$.

in the natural frequency of plates 1 and 2 as their thicknesses change. Before discussing the effect of mechanical-acoustic coupling on the electricity generation efficiency, we must make sure of the efficiency $P_{e m 1}$ under only the plate vibration without coupling.

Figure 12 shows, theoretically and experimentally, such an efficiency as functions of the thickness $h_{c}$. In the theoretical results, $T_{n}=10^{8}$ and $R_{n}=10^{1}$ are adopted as the support condition. Since the electric power caused by the electricity generation and the mechanical power supplied to the plate (i.e., the input and output power) depend on those of the flexural displacement and vibration velocity, decreases in the flexural rigidity from thinning the plate and increases in the natural frequency from thickening the plate are factors to increase both powers. In general, the flexural displacement is inversely proportional to $h_{c}{ }^{3}$, and the natural frequency is directly proportional to $h_{c}$, so that $P_{e m 1}$ is inversely proportional to $h_{c}{ }^{2}$ if the point force is constant in the entire range of $h_{c}$.

The same figure also indicates the total $P_{e m}$ with mechanical-acoustic coupling. $P_{e m}$ increases greatly with $h_{c}$ up to approximately $h_{c}=4 \mathrm{~mm}$, so that the efficiency exceeds that in the electricity generation without coupling and the difference between both efficiencies is expanded with increasing $h_{c}$. On the other hand, the theoretical $P_{e m}$ decreases with increasing $h_{c}$ in subsequent variations. The maximum value reaches approximately $14 \%$, which appears at approximately $h_{c}=4 \mathrm{~mm}$. These results are derived from specific conditions in this study such as the dimensions, mechanical, and electric properties, and so on of the plate and the piezoelectric element and its electrode, so that we cannot conclude that those results are generalized. However, it is remarkable that the experimental results demonstrate the validity of the theoretical analysis and verify the usefulness of this electricity generation system. 


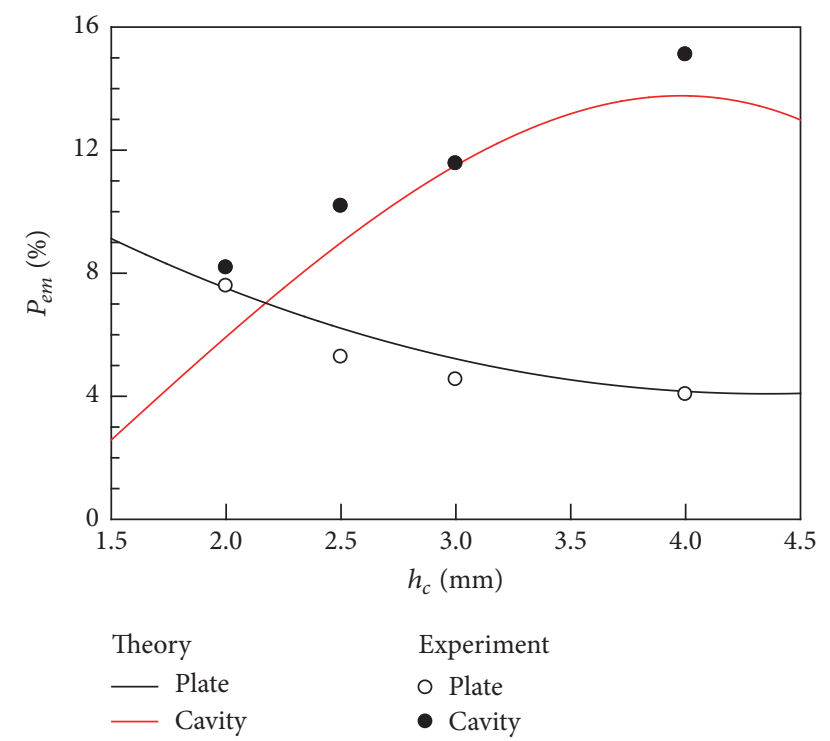

FIGURE 12: Variations in electricity generation efficiency with plate thickness.

\section{Conclusion}

We improved the electricity generation characteristics derived from the vibration of a plate with a piezoelectric element installed at its center. The cylinder had such plates at both ends. One side of each plate was subjected to a harmonic point force. We used a mechanical-acoustic coupling between the plate vibrations and internal sound field. In this study, the effect of mechanical-acoustic coupling on energyharvesting was estimated theoretically and experimentally from the electricity generation efficiency. In particular, electromechanical-acoustic coupling was taken into consideration in the theoretical study.

For electricity generation caused only by the plate vibration without mechanical-acoustic coupling, the electric power and electricity generation efficiency are maximized at the natural frequency of the generation system and significantly exceed those in the frequency range except for the natural frequency region. However, the natural frequency of the system, in which the stick to apply the mechanical power to the plate is contained, is somewhat lower than that of the plate.

With respect to the electricity generation system using mechanical-acoustic coupling, it is confirmed experimentally that the electric power of the nonexcitation side increases significantly in comparison with that of the excitation side, in which the plate is excited by the point force of its natural frequency. The theoretical and experimental studies reveal that the electricity generation efficiency is improved by using mechanical-acoustic coupling in comparison with that of the electricity generation caused only by the plate vibration without coupling.

\section{Competing Interests}

The authors declare that they have no competing interests.

\section{Acknowledgments}

This work was partially supported by JSPS KAKENHI Grant no. $15 \mathrm{~K} 05874$.

\section{References}

[1] S. R. Anton and H. A. Sodano, "A review of power harvesting using piezoelectric materials (2003-2006)," Smart Materials and Structures, vol. 16, no. 3, pp. R1-R21, 2007.

[2] K. Yamada, H. Matsuhisa, and H. Utsuno, "Improvement of efficiency of piezoelectric element attached to beam based on mechanical impedance matching," Journal of Sound and Vibration, vol. 333, no. 1, pp. 52-79, 2014.

[3] M. Piñeirua, O. Doaré, and S. Michelin, "Influence and optimization of the electrodes position in a piezoelectric energy harvesting flag," Journal of Sound and Vibration, vol. 346, no. 1, pp. 200-215, 2015.

[4] J. Pan and D. A. Bies, "The effect of fluid-structural coupling on sound waves in an enclosure-theoretical part," Journal of the Acoustical Society of America, vol. 87, no. 2, pp. 691-707, 1990.

[5] J. Pan and D. A. Bies, "The effect of fluid-structural coupling on sound waves in an enclosure-experimental part," Journal of the Acoustical Society of America, vol. 87, no. 2, pp. 708-717, 1990.

[6] L. Cheng and J. Nicolas, "Radiation of sound into a cylindrical enclosure from a point-driven end plate with general boundary conditions," Journal of the Acoustical Society of America, vol. 91, no. 3, pp. 1504-1513, 1992.

[7] L. Cheng, "Fluid-structural coupling of a plate-ended cylindrical shell: vibration and internal sound field," Journal of Sound and Vibration, vol. 174, no. 5, pp. 641-654, 1994.

[8] H. Moriyama and Y. Tabei, "Acoustic characteristics inside cylindrical structure with end plates excited at different frequencies," Journal of Visualization, vol. 7, no. 1, pp. 93-101, 2004.

[9] H. Moriyama, Y. Tabei, and N. Masuda, "Acoustic characteristics of a sound field inside a cylindrical structure with excited end plates: influence of excitation position on vibro-acoustic coupling," Acoustical Science and Technology, vol. 26, no. 6, pp. 477-485, 2005.

[10] H. Moriyama, H. Tsuchiya, and Y. Oshinoya, "Energy harvesting with piezoelectric element using vibroacoustic coupling phenomenon," Advances in Acoustics and Vibration, vol. 2013, Article ID 126035, 11 pages, 2013.

[11] A. Kojima, H. Moriyama, and Y. Oshinoya, "Vibroacoustic coupling of cylindrical enclosure with excited end plates," Acoustical Science and Technology, vol. 33, no. 3, pp. 180-189, 2012.

[12] W. Larbi, J.-F. Deü, and R. Ohayon, "Finite element formulation of smart piezoelectric composite plates coupled with acoustic fluid," Composite Structures, vol. 94, no. 2, pp. 501-509, 2012.

[13] W. Larbi, J.-F. Deü, R. Ohayon, and R. Sampaio, "Coupled FEM/BEM for control of noise radiation and sound transmission using piezoelectric shunt damping," Applied Acoustics, vol. 86, pp. 146-153, 2014.

[14] P. M. Morse and K. U. Ingard, Theoretical Acoustics, McGrawHill, New York, NY, USA, 1968. 


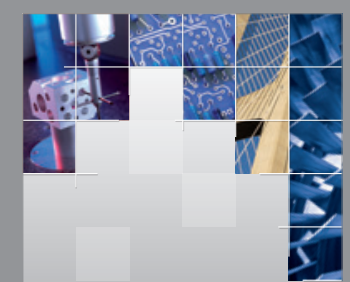

\section{Enfincering}
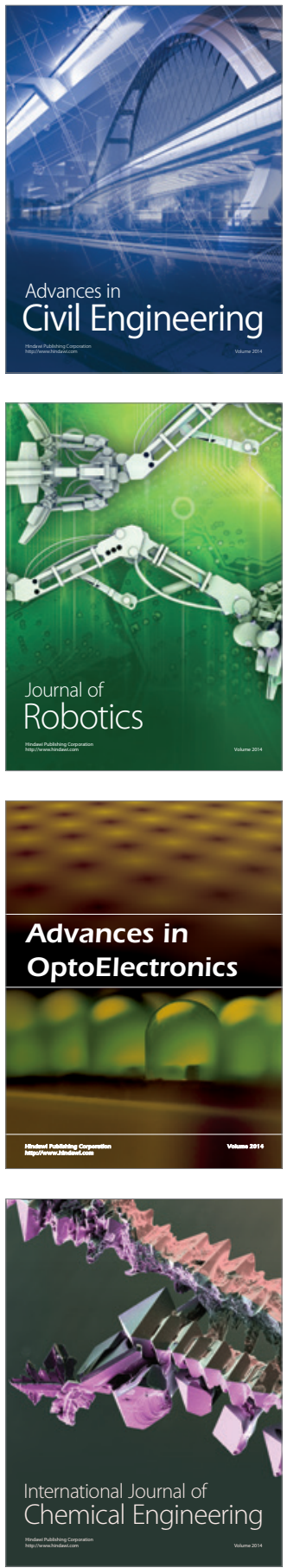

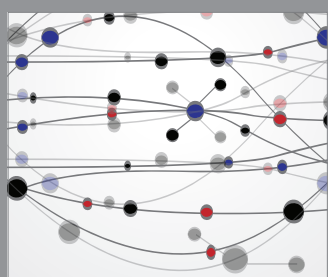

The Scientific World Journal

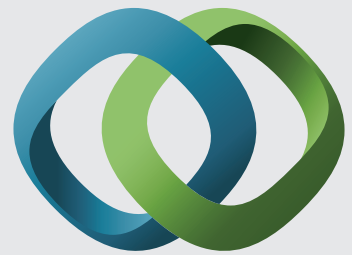

\section{Hindawi}

Submit your manuscripts at

http://www.hindawi.com
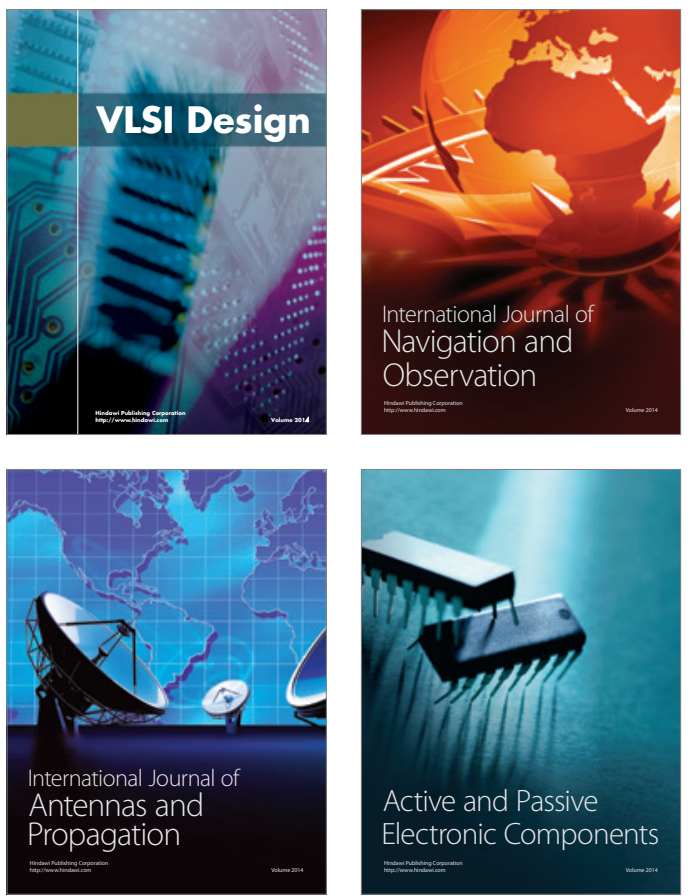
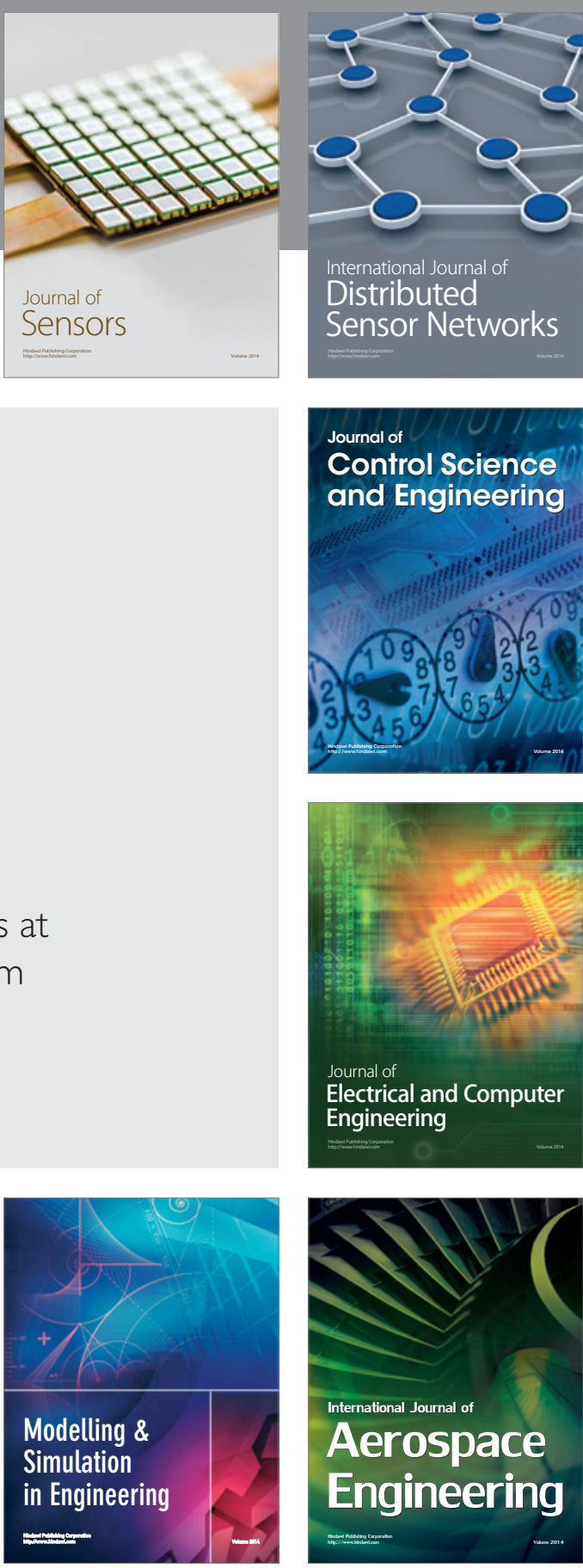

International Journal of

Distributed

Sensor Networks

Journal of

Control Science

and Engineering
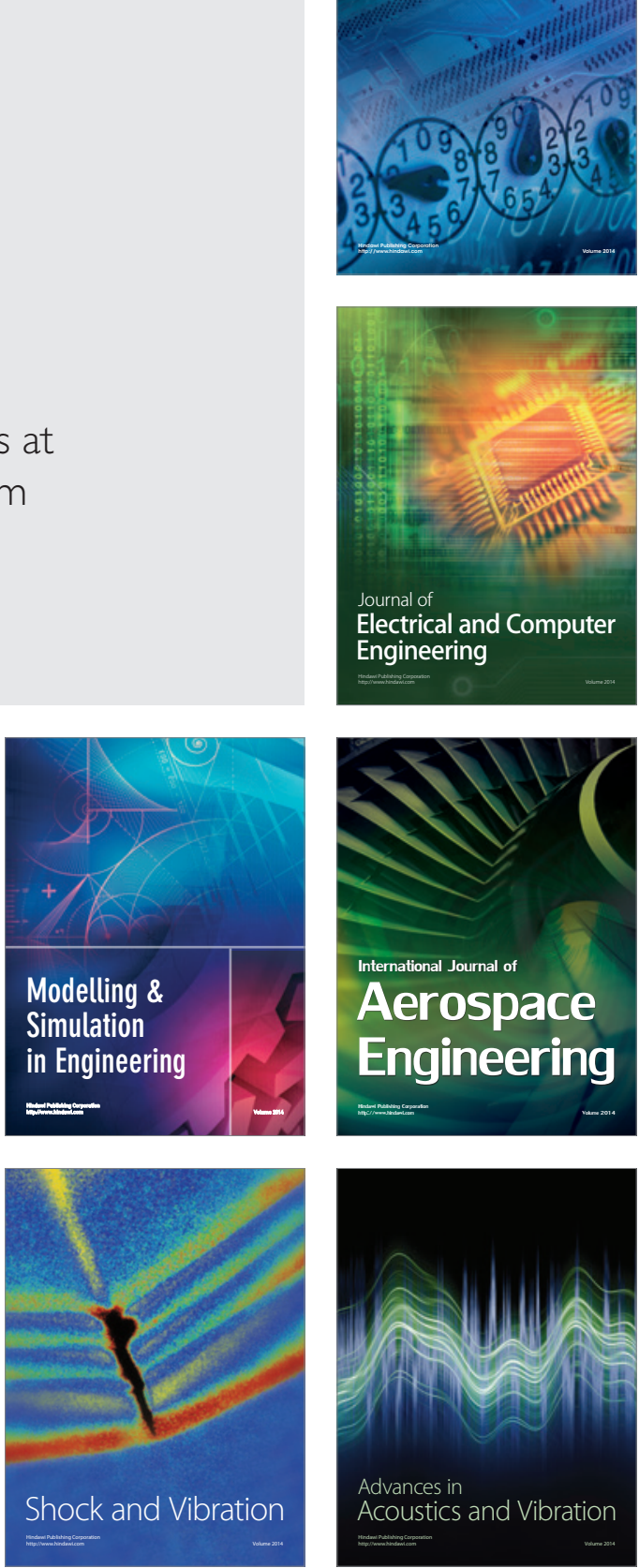